\title{
Optical, Thermal and Nonlinear Properties of Tristhiourea Lanthanum Nitrate
}

\author{
V. S. Kumar1, R. S. Sundararajan² \\ ${ }^{1}$ Department of Physics, Swami Dayananda College of Arts and Science, Manjakkudi, Tamilnadu, India \\ ${ }^{2}$ Department of Physics, Government Arts College (Autonomous), Kumbakonam, Tamilnadu, India \\ Email: shanmugavsk.2010@gmail.com
}

Received 25 February 2015; accepted 27 March 2015; published 31 March 2015

Copyright (C) 2015 by authors and Scientific Research Publishing Inc.

This work is licensed under the Creative Commons Attribution International License (CC BY).

http://creativecommons.org/licenses/by/4.0/

(c) (i) Open Access

\begin{abstract}
The single crystals of tristhiourea lanthanum nitrate (TTLaN) have been grown by solution growth using slow evaporation technique. The optical study revealed that the crystal has high transmission with lower cut-off wavelength of $235.78 \mathrm{~nm}$. The EDAX analysis confirmed the grown compound elements. The presence of functional groups was identified through Fourier transforminfrared technique. The thermal stability of the crystal was determined from thermo gravimetric and differential thermal analysis curve. The second harmonic generation behavior of TTLaN crystal was tested by Kurtz-Perry powder technique. This single novel semiorganic crystal of TTLaN showed very good optical transmission property, high thermal stability and large non-linearity nature.
\end{abstract}

\section{Keywords}

Nonlinear Optical, Tristhiourea Lanthanum Nitrate, Second Harmonic Generation, Thermo Gravimetric Analysis, Differential Thermal Analysis

\section{Introduction}

A great recital optic switching aspect for optical information and telecommunication dispensation is based on non-linear optical (NLO) materials. A selection of such materials has been scrutinized. Normally, many organic crystals reveal enormously high nonlinearities much better than inorganic crystals, but they have thermal wobbling nature. Hence current research is paying attention on semi-organic materials due to their high laser damage threshold, good mechanical and thermal stability with nonlinear properties [1] [2]. At present, metal complexes of thiourea and its derivatives have been investigated. Thiourea (TU) is an organosulfur compound with the formula $\mathrm{SC}\left(\mathrm{NH}_{2}\right)_{2}$. It is structurally similar to urea, except that the oxygen atom is replaced by a sulfur atom, but 
the properties of urea and thiourea differ significantly. Thiourea is used in many industrial applications and as a chemical intermediate, in metal processing, plating and in photo processing. It has a wide range of uses, such as producing and modifying textile, dyeing auxiliaries, and synthetic resins in image reproduction in the production of pharmaceuticals and industrial cleaning agents for etching process in electroplating and also as an antioxidant. It is also a centrosymmetric molecule; if coordinated with metal ions, it becomes noncentrosymmetric materials showing nonlinear activity. In the present work, an attempt has been made to grow and characterize single novel crystals of tristhiourea lanthanum nitrate (TTLaN) where the lanthanum belongs to rare earth group.

\section{Experimental}

High purity salts (99.9\%) purchased from E-Merck were used for the crystal growth. Single crystals of tristhiourea lanthanum nitrate (TTLaN) were grown at room temperature by solution growth using slow evaporation method, of an aqueous solution containing thiourea and lanthanum nitrate in stoichiometric ratio 3:1 as per the reaction. The temperature of water in the bath was controlled digitally by using microprocessor. Variation in temperature of the bath can be tuned to an accuracy of $\pm 0.1^{\circ} \mathrm{C}$.

$$
3 \mathrm{CS}\left[\mathrm{NH}_{2}\right]_{2}+\mathrm{La}\left(\mathrm{NO}_{3}\right)_{3} \cdot 6 \mathrm{H}_{2} \mathrm{O} \rightarrow \mathrm{La}\left[\mathrm{CS}\left(\mathrm{NH}_{2}\right)_{2}\right]_{3} \mathrm{NO}_{3}
$$

Optically good quality crystals of the title compound were harvested in span of 40 days. The photograph of the TTLaN crystal is shown in Figure 1.

\section{Characterization}

Fourier transform infrared spectrum was recorded by the KBr pellet technique using a Bruker $66 \mathrm{~V}$ FTIR spectrometer to confirm the vibrational structure of the crystalline compound with scanning range of wave number $400-4000 \mathrm{~cm}^{-1}$. UV-Vis spectrum was recorded in the range of $200-2000 \mathrm{~nm}$ using VARIAN CARY 5E spectrometer. Micro hardness studies were carried out at room temperature using a micro hardness tester, fitted with a diamond indenter using Vicker's hardness method. The surface morphology and elemental analysis were studied using TESCAN SEM-VEGA III and CONTEXT softwares respectively. Thermal behavior of the grown sample was studied using instrument NETZSCH STA 449F3 thermal analyzer. The NLO property of TTLaN crystal was tested by Kurtz powder SHG (Second Harmonic Generation) test using Nd:YAG laser (1064 nm).

\section{Results and Discussion}

\subsection{Fourier Transform Infrared Studies}

The infrared spectrum of the grown crystal has been taken in the range of $400-4000 \mathrm{~cm}^{-1}$. The sample is made as a pellet by using $\mathrm{KBr}$. The FTIR spectrum of tristhiourea Lanthanum nitrate (TTLaN) is shown in Figure 2. The spectrum shows the presence of all the functional groups in TTLaN crystal and summarized in Table 1. In

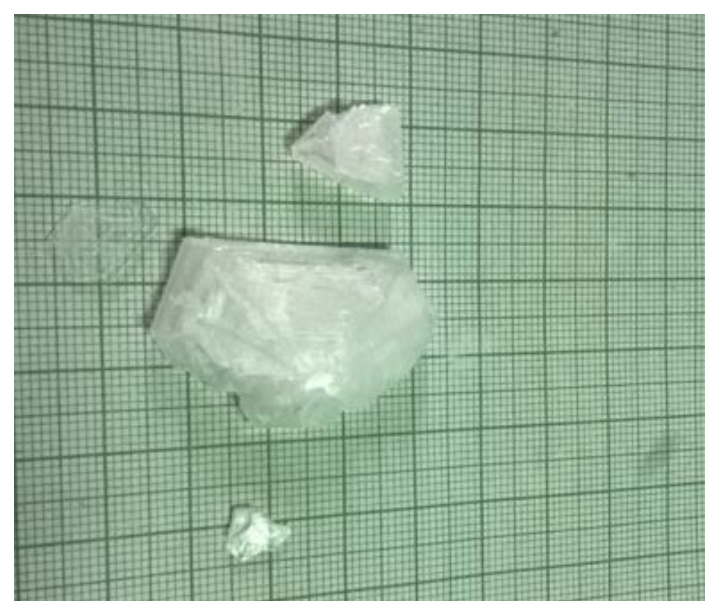

Figure 1. Photograph of the grown TTLaN crystals. 


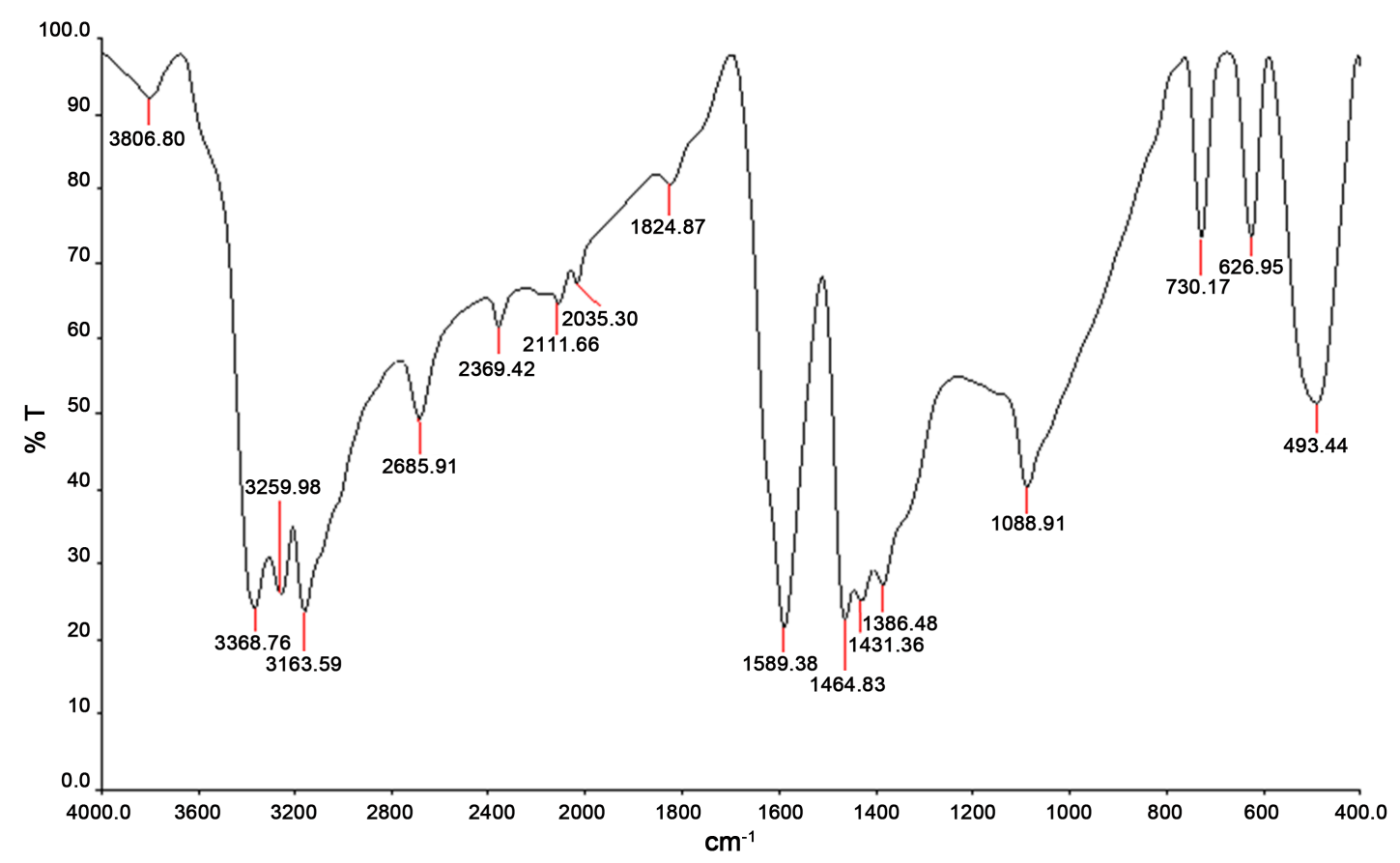

Figure 2. FTIR spectrum of TTLaN.

Table 1. IR vibrational wave numbers and assignments of thiourea and TTLaN.

\begin{tabular}{ccc}
\hline $\mathrm{TU} \mathrm{cm}^{-1}$ & $\mathrm{TTLaN} \mathrm{cm}^{-1}$ & Assignment Modes \\
3380 & 3806.80 & $v(\mathrm{~N}-\mathrm{H})$ \\
3279 & 3259.98 & $v(\mathrm{~N}-\mathrm{H})$ \\
3190 & ---- & $v(\mathrm{~N}-\mathrm{H})$ \\
3177 & 3163.59 & $v(\mathrm{~N}-\mathrm{H})$ \\
1620 & ---- & $\delta(\mathrm{N}-\mathrm{H})$ \\
---- & 1589.38 & $\delta(\mathrm{N}-\mathrm{H})$ \\
1477 & 1464.83 & $v(\mathrm{~N}-\mathrm{C}-\mathrm{N})$ \\
1414 & 1431.36 & $v(\mathrm{C}-\mathrm{S})$ \\
1082 & 1088.91 & $\rho \mathrm{NH} 2$ \\
730 & 730.17 & $v(\mathrm{C}-\mathrm{N})$ \\
---- & 626.95 & $v(\mathrm{C}-\mathrm{N})$ \\
494 & 493.44 & $\delta(\mathrm{N}-\mathrm{C}-\mathrm{S})$ \\
\hline
\end{tabular}

$\nu$-stretching; $\rho$-rocking; $\delta$-deformation.

the FT-IR spectrum Figure 2 of grown crystal of TTLaN, the lower peaks are observed according to the wave number ranging from $400-4000 \mathrm{~cm}^{-1}$. The Lanthanum nitrate peaks are compared with thiourea peaks for the corresponding assignment Table 1. The NH stretching vibrational bands of $\mathrm{NH}_{2}$ were observed at $3806.8 \mathrm{~cm}^{-1}$, $3368.76 \mathrm{~cm}^{-1}$, $3259.98 \mathrm{~cm}^{-1}$ and $3163.59 \mathrm{~cm}^{-1}$, whereas it was observed at $3380 \mathrm{~cm}^{-1}$ and $3177 \mathrm{~cm}^{-1}$ and 3190 $\mathrm{cm}^{-1}$ for thiourea respectively. The IR band for $\mathrm{NH}_{2}$ rocking was observed at $1620 \mathrm{~cm}^{-1}$ for thiourea. The N-C-N stretching vibration was found at $1464.83 \mathrm{~cm}^{-1}$ in the observed IR spectrum, whereas it was observed at $1477 \mathrm{~cm}^{-1}$ for thiourea. The C-S stretching vibration was found at $1431.36 \mathrm{~cm}^{-1}$ for TTLaN and for thiourea at1414 $\mathrm{cm}^{-1}$ in the observed IR spectrum. The IR band for $\mathrm{NH}_{2}$ rocking was observed at $1081 \mathrm{~cm}^{-1}$, whereas it 
was observed at $1088.91 \mathrm{~cm}^{-1}$ for thiourea. The symmetric stretching band is recorded at $730.17 \mathrm{~cm}^{-1}$, whereas it was recorded at $730 \mathrm{~cm}^{-1}$ for TU. The IR band of N-C-S bending vibration is observed at $493.44 \mathrm{~cm}^{-1}$, whereas it was observed at $494 \mathrm{~cm}^{-1}$ for TU. This study Table 1 reveals that there is a good agreement between thiourea and thiourea present in TTLaN for various FTIR assignments.

\subsection{Optical Transmission Studies}

Figure 3 shows the optical transmission spectrum TTLaN single crystals. From the spectrum, it is evident that the TTLaN crystal has a very lower UV cut-off wavelength of $235.78 \mathrm{~nm}$, along with a large transmission window in the entire visible region. This makes it suitable for more optoelectronic applications [3].

\subsection{Hardness Studies}

Micro hardness studies were carried out at room temperature using a micro hardness tester, fitted with a diamond indenter. The diagonal lengths of indented impressions obtained at various loads were measured using a micrometer eyepiece. The Vicker's hardness number $\mathrm{H}_{\mathrm{v}}=1.8544 \mathrm{P} / \mathrm{d}^{2} \mathrm{Kg} / \mathrm{mm}^{2}$ where $\mathrm{P}$ is the applied load in $\mathrm{Kg}$ and $\mathrm{d}$ is the average diagonal length of the indentation mark in mm [4]. Figure 4 shows a graph plotted between $\log \mathrm{P}$ and logd. From this observation, the material has a higher hardness property and is useful for nonlinear optical applications. Figure 5 shows the variation of hardness number with three different loads. $\mathrm{The}_{\mathrm{v}}$

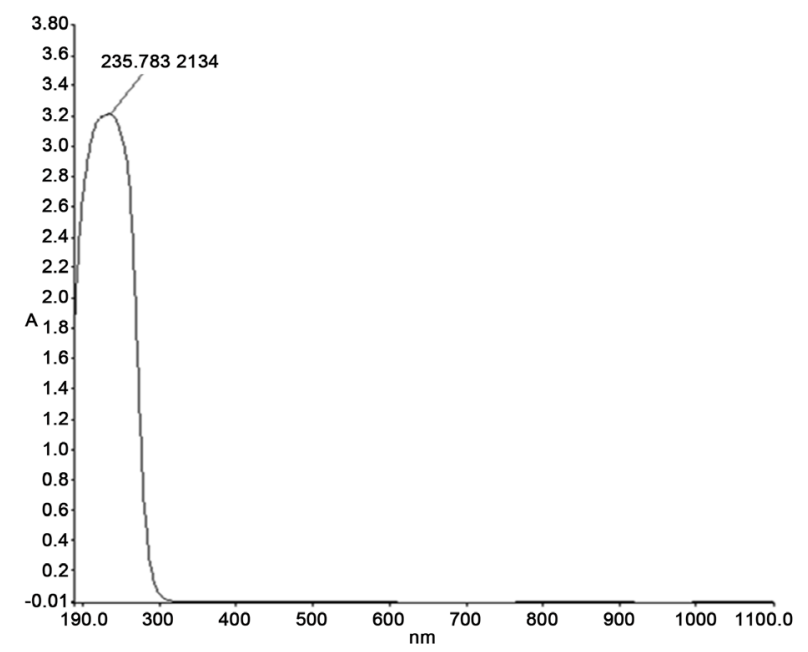

(a)

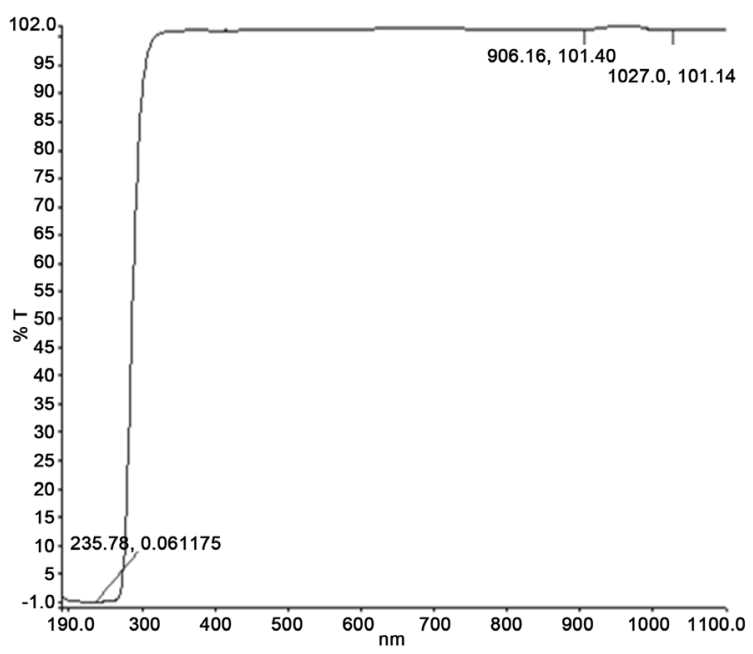

(b)

Figure 3. UV-Vis NIR spectrum of TTLaN. (a) Absorbance spectrum of TTLaN; (b) Transmission spectrum of TTLaN.

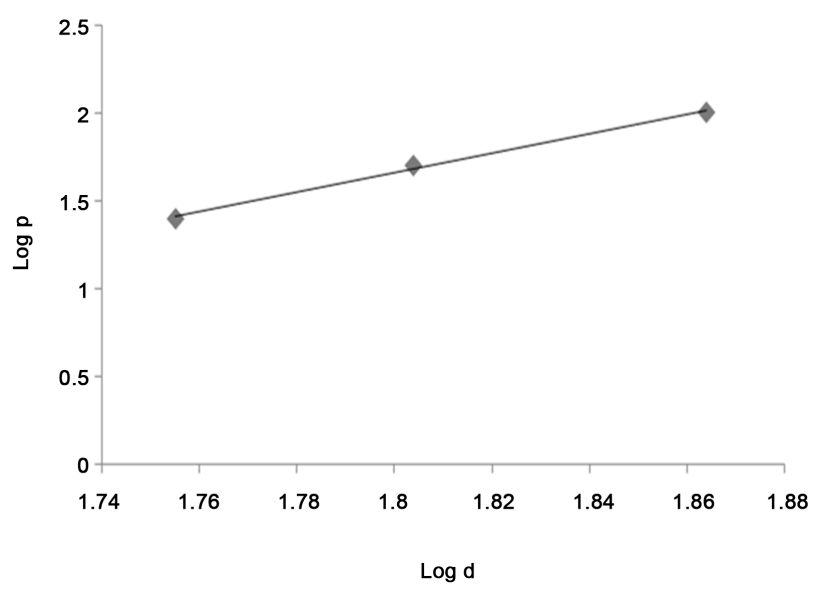

Figure 4. Graph between logP vs logd. 


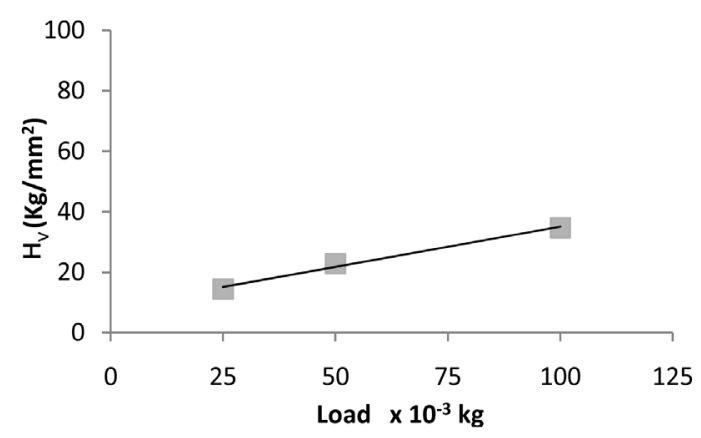

Figure 5. Graph between $H_{v}$ vs load.

increases with increase in applied load. The micro hardness study reveals that the grown crystals are mechanically hard. Higher the hardness values, greater the stress required to form dislocation, thus confirming greater crystalline perfection.

\subsection{Surface Morphology Studies and Elemental Analysis}

Figure 6(a) and Figure 6(b) shows the scanning electron micrographs of TTLaN crystals using TESCAN SEM. The image implies a mixed dense and columnar structure. The SEM observations show a toothed structure on the top layer with fine vertical line patterns. At higher magnification it is observed as the bulk growth. Figure 7 confirmed the elements present in the title compound. The presence of $\mathrm{C}, \mathrm{N}, \mathrm{La}, \mathrm{S}, \mathrm{O}$ peaks in EDAX spectrum confirmed the presence of grown tristhiourea lanthanum nitrate.

\subsection{Thermal Studies}

The thermal characteristics of TTLaN, differential thermal analysis (DTA) and thermo gravimetric analysis (TGA) were carried out by the instrument NETZSCH STA 449F3 simultaneously. The sample was heated at a rate of $20^{\circ} \mathrm{C} / \mathrm{min}$ in the region $0^{\circ} \mathrm{C}$ to $1000^{\circ} \mathrm{C}$ to testify the weight loss and thermal stability. Figure 8 shows the thermo grams illustrating simultaneously recorded TGA and DTA. The irreversible endothermic transition at $240^{\circ} \mathrm{C}$ where the decomposition starts. The material is fully decomposed at $275^{\circ} \mathrm{C}$. The weight loss curve is very sharp and it starts at $240^{\circ} \mathrm{C}$ and ends at $325^{\circ} \mathrm{C}$. This weight loss is due to the liberation of volatile substances. The sharpness of the endothermic peak shows good degree of crystallinity of the grown crystal. The peak at $325^{\circ} \mathrm{C}$ indicates a phase transition from liquid to vapor state as evidence from the loss of weight in the TG curve.

\subsection{NLO Studies}

The second harmonic generation SHG test on the TTLaN crystal was performed by Kurtz powder SHG method [5]. The powdered sample of TTLaN crystal was illuminated using the fundamental beam of $1064 \mathrm{~nm}$ from Q-switched Nd:YAG laser. The input energy incident on the sample was $0.68 \mathrm{~J}$, an energy level optimized to cause no chemical decomposition of the sample. The output energy of the sample is $4.3 \mathrm{~mJ}$. The KDP reference energy is $8.8 \mathrm{~mJ}$. Hence, the title compound has good nonlinearity.

\section{Conclusion}

Single crystals of tristhiourea lanthanum nitrate-a new semi-organic NLO material have been grown from aqueous solution. The optical transmission shows lower cut-off region of the crystal at $235.78 \mathrm{~nm}$. The crystal has sufficient transmission also in the entire visible region. The element analysis confirms the presence of lanthanum, nitrate and thiourea atoms. FTIR analysis confirms the presence of characteristic functional groups. The purity of the crystal is confirmed from the sharpness of the endothermic peaks. The SHG confirms the NLO property of the crystal. The mechanical strength was proved by the micro-hardness study. Thus, TTLaN crystals have good optical transmission property, high thermal stability and large non-linearity nature.

\section{Acknowledgements}

The authors are thankful to Annamalai University, ACIC, St. Joseph’s College for the thermal analysis and 


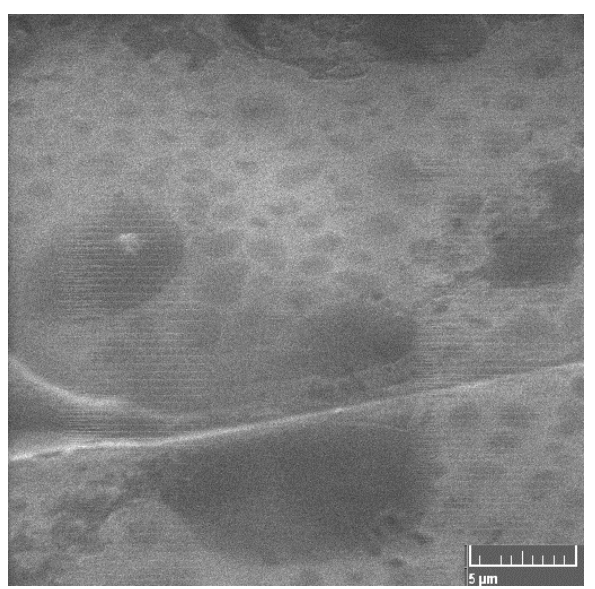

(a)

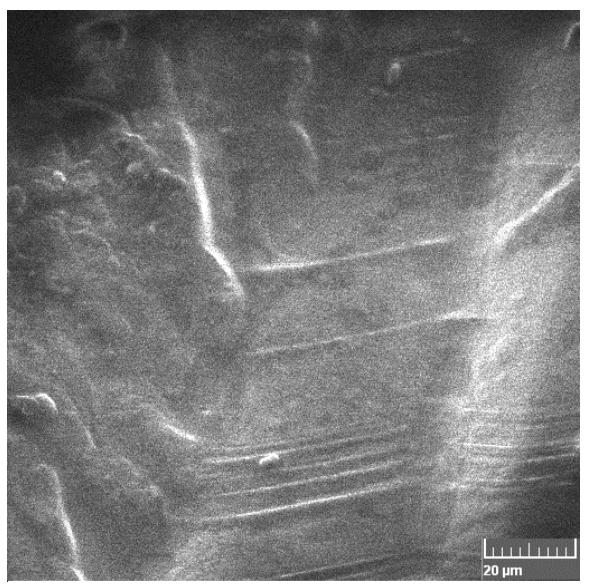

(b)

Figure 6. SEM images of TTLaN crystals.

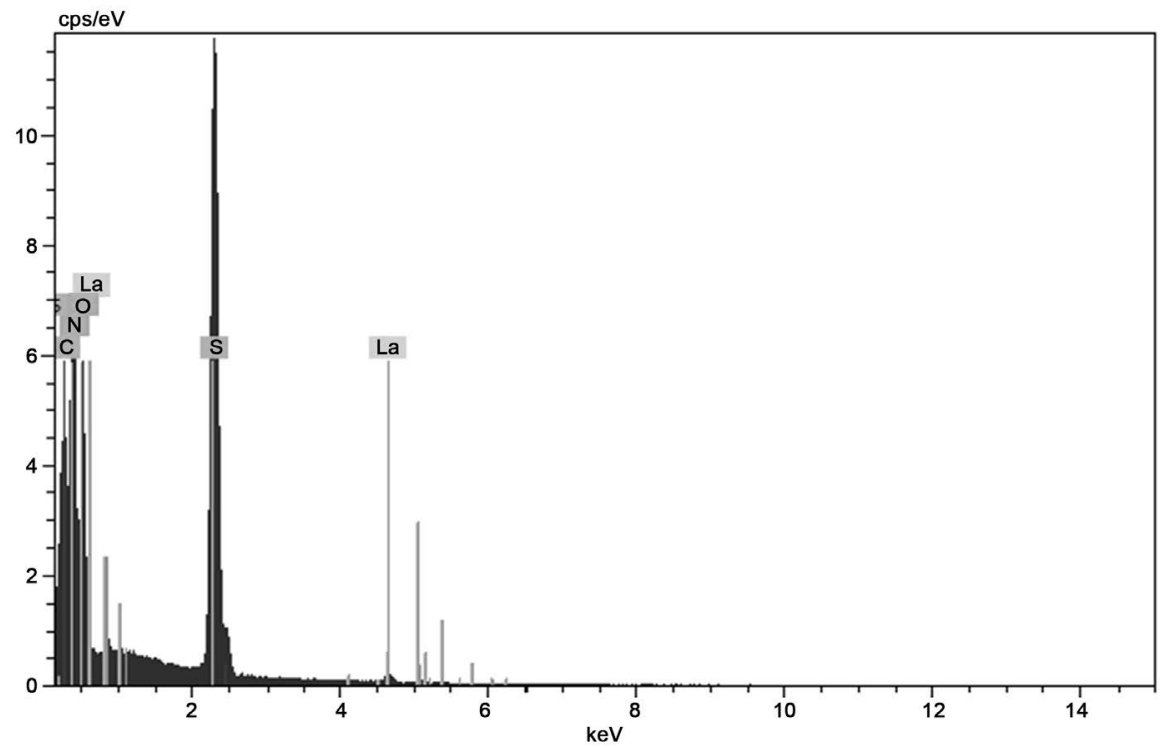

Figure 7. EDAX image of TTLaN.

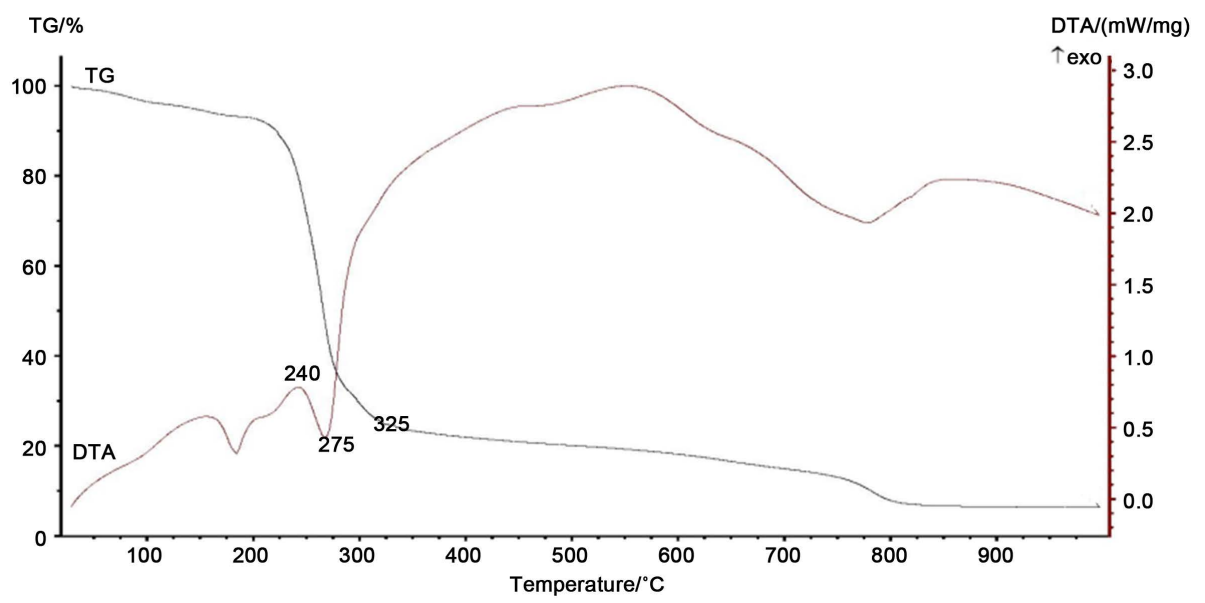

Figure 8. TG-DTA curve of TTLaN. 
spectral studies respectively, B. S. Abdul Rahuman University for SHG, CECRI, Karaikudi for elemental analysis and SEM images.

\section{References}

[1] Jonie Varjula, A., Vesta, C., Justin Raj, C., Dinakaran, S., Ramanand, A. and Jerome Das, S. (2007) Growth and Characterization of a New Semi-Organic Nonlinear Optical Sodium Paranitrophenolate Paranitrophenol Dehydrate Single Crystal. Materials Letters, 61, 5053-5055. http://dx.doi.org/10.1016/j.matlet.2007.04.012

[2] Rajesh, N.P., Kannan, V., Ashok, M., Sivaji, K., Santhana Raghavan, P. and Ramaswamy, P. (2004) A New Nonlinear Optical Semi-Organic Material: Cadmium Thiourea Acetate. Journal of Crystal Growth, 262, 561-566. http://dx.doi.org/10.1016/j.jcrysgro.2003.10.064

[3] Sundararajan, R.S., Senthilkumar, M. and Ramchandraraja, C. (2013) Growth and Characterization of Bisthiourea Nickel Bromide-New Semiorganic Nonlinear Optical Crystal. Journal of Crystallization Process and Technology, 3, 56-59. http://dx.doi.org/10.4236/jcpt.2013.32008

[4] Mott, B.W. (1956) Micro-Indentation Hardness Testing. Butterworths, London, 206.

[5] Kurtz, S.K. and Perry, T.T. (1968) A Powder Technique for the Evaluation of Nonlinear Optical Materials. Journal of Applied Physics, 39, 3798-3813. http://dx.doi.org/10.1063/1.1656857 